\title{
Postural Stability Challenges in Narrow Walking after Traumatic Brain Injury
}

\author{
Langenderfer Joseph E., ${ }^{1, *}$ Mara R. Thompson², Ustinova Ksenia I. ${ }^{2}$ \\ ${ }^{1}$ School of Engineering and Technology, Central Michigan University, Mount Pleasant, 48859, Michigan, United States \\ ${ }^{2}$ Department of Physical Therapy, Central Michigan University, Mount Pleasant, 48859, Michigan, United States
}

Received June 8, 2021; Revised July 8, 2021; Accepted August 22, 2021

\begin{abstract}
Cite This Paper in the following Citation Styles
(a): [1] Langenderfer Joseph E., Mara R. Thompson, Ustinova Ksenia I. , "Postural Stability Challenges in Narrow Walking after Traumatic Brain Injury," International Journal of Human Movement and Sports Sciences, Vol. 9, No. 5, pp. 948 - 954, 2021. DOI: 10.13189/saj.2021.090516.
\end{abstract}

(b): Langenderfer Joseph E., Mara R. Thompson, Ustinova Ksenia I. (2021). Postural Stability Challenges in Narrow Walking after Traumatic Brain Injury. International Journal of Human Movement and Sports Sciences, 9(5), 948 - 954. DOI: 10.13189/saj.2021.090516.

Copyright $\odot 2021$ by authors, all rights reserved. Authors agree that this article remains permanently open access under the terms of the Creative Commons Attribution License 4.0 International License

\begin{abstract}
The aim of this study was to evaluate the predictability and complexity of gait under preferred and narrow walking conditions for groups of TBI and age matched control subjects in order to determine if non-linear measures may be appropriate for identifying gait deviations not detected with more routine techniques. Ten participants from each group walked at preferred gait and with a narrow base of support. Center of mass motion was calculated from marker motion recorded with motion capture. Measures of predictability and complexity of center of mass motion were calculated with Recurrence Quantification Analysis. Walking with a narrow base of support resulted in a less predictable center of mass motion (reduced \%Determinism) for TBI and control groups $(\mathrm{p}=0.0019$ and $\mathrm{p}=0.0008$, respectively), but no difference was found between groups. For the TBI group, there was a significant decrease in complexity of center of mass motion (lower Entropy, $\mathrm{p}=0.0038$ ) with narrow base of support compared to preferred walking, but no difference in Entropy was found between groups. These results suggest non-linear measures determined using techniques such as Recurrence Quantification Analysis might have potential to be valid and reliable measurement techniques for predicting gait deviations in future studies.
\end{abstract}

Keywords RQA, TBI, Non-Linear, Recurrence, Quantification, Analysis

\section{Introduction}

Observational and instrumental gait analysis is challenging in individuals with post-traumatic brain injury (TBI) when compared to other clinically encountered deficits. Post-TBI sensorimotor impairments do not have a specific pattern of manifestation(s), as compared to gait deviations after spinal cord injury or Parkinson's disease. Furthermore, for TBI the compensations for gait impairments can be even more variable. Consequently, post-TBI gait deviations, especially when well compensated, can be quite hidden, thus making recognition of particular deviations quite difficult.

Gait analysis has significantly improved with development of instrumented techniques and algorithms for measuring and interpreting the most typical abnormalities. To date, a comprehensive list of kinetic and kinematic gait deviations from norms is well known. The deviations can include, but are not limited to, changes in spatial characteristics, timing of gait phases and sub-phases, range of motions in major joints, exerted joint torques, center of mass (COM) displacements and even more, e.g. summarized in works by $[1,2]$. Paradoxically, an expansion of the gait measure list sometimes does not increase sensitivity to detect small changes in gait performance. This limitation is because most gait characteristics are derivatives of the same or similar measures, e.g. spatial displacement per time, and therefore naturally correlate one with another. Detection of small gait deviations become even more problematic in 
individuals with relatively insignificant clinical impairments, as for example post mild TBI injury. When undetected, but somehow compensated and therefore not addressed by rehabilitation, respectively, these hidden deviations may manifest at an inappropriate time while walking, cause unexpected perturbations to gait, and thus increase the risk of fall and injury. In this regard, the development of more sensitive measures for detecting even small or hidden gait deviations is important.

The following example can illustrate the above statement regarding need for more sensitive measures. In our previous experiment [3], gait patterns in individuals with TBI were compared with those of healthy participants in several walking conditions. While some gait characteristics differed, many were similar for the two groups. Most surprisingly was the fact that postural stability, analyzed with the COM displacement as a common measure, did not show a between-group difference, despite the fact that observationally individuals with TBI were much less stable than healthy participants and required some guarding to ensure a safe gait. This observation was the genesis for utilizing a different approach of analysing postural stability, rather than using traditional COM or COP measures.

Non-linear analysis has been useful in identification of pre-clinical, but potentially pathological, factors associated with pain, aging and disease [4, 5]. For standing postural control, non-linear methods have shown greater sensitivity than traditional measures in identifying differences following concussion [6-8]. In similar manner, non-linear dynamic analysis may be more sensitive, and thus useful, in identifying specific aspects of a stable and functional gait. Recurrence Quantification Analysis (RQA) is a non-linear technique for describing the structure of variability in terms of how a system varies behavior in response to environmental requirements. RQA has been used to gain increased understanding of biological system variability including postural control and gait under unimpaired conditions [9-11], and for physiologically compromised situations [12-14]. However, little information exists to explain how TBI affects the pattern of gait variability.

Therefore, the purpose of this study was to use RQA to examine gait stability while walking at preferred gait and with narrow base of support in individuals with TBI and a control group of subjects. RQA measured the predictability and complexity of COM motion variability in order to determine the appropriateness of RQA for assessing differences in gait stability. Overall, it was hypothesized that RQA measure would provide a more comprehensive assessment of postural stability while walking, and therefore may allow for detection of hidden postural stability deficits present in individuals with TBI while walking. Specifically, TBI was expected to result in less repeatable and less predictable COM motion during the intervention of narrow base of support walking compared to unimpaired Control participants. Secondly, it was hypothesized that narrow walking would cause TBI subjects to alter gait resulting in a less repeatable and less complex COM motion.

\section{Materials and Methods}

\subsection{Participants}

Ten participants ( $3 \mathrm{~F}, 7 \mathrm{M}$, mean age $45.2 \pm 12.7$ (SD) years) with TBI (average time from TBI of $10.3 \pm 6.3$ years) were recruited from local community outpatient clinics and support groups. Informed consent was granted by all participants as required by the Institutional Review Board (IRB). The criteria for inclusion in this study was: a TBI sustained over 6 months previously, ability to walk independently without assistive device for $30 \mathrm{~m}$, full or nearly-full range of motion in major body joints, ability to follow simple instructions and normal or corrected-to-normal vision. TBI participants presented with some amount of ataxia and postural and gait abnormalities (Table 1), according to standard clinical tests and ranges of scores: (a) Ataxia Test by Klockgether [15]: 2-18 (mean \pm SD: $7.9 \pm 6.1), 35$ indicates severe ataxia; (b) Berg Balance test (BBS) [16]: 45-54 (51.0 \pm 3.6), 45 indicates an increased risk of fall; and (c) Functional Gait Assessment Test (FGA) [17] 14-27 (mean \pm SD: $22.8 \pm 4.3$ ), 22 indicates an increased risk of fall. The degree of brain injury was determined based on loss of consciousness (LOS) duration [18]. By this classification, LOS for < 30 minutes indicates mild TBI; > 30 minutes and $<24$ hours - moderate TBI, and > 24 hours - severe TBI. Of the 10 participants with TBI, 2 individuals (\#1-2 Table 1) had a moderate and 5 individuals (\#3-7 Table 1) mild TBI. The duration of LOS was not available for participants \#8-10. In addition to the TBI group, there were 10 age-matched control participants. 
Table 1. Demographics and clinical evaluation scores of TBI participants

\begin{tabular}{|c|c|c|c|c|c|c|}
\hline Subject & $\begin{array}{l}\text { Gender } \\
(\mathrm{M} / \mathrm{F})\end{array}$ & $\begin{array}{c}\text { Age } \\
\text { (years) }\end{array}$ & $\begin{array}{c}\begin{array}{c}\text { TBI onset } \\
\text { (years) }\end{array} \\
\end{array}$ & $\begin{array}{c}\text { Ataxia } \\
(0-35 \mathrm{pts}) \\
\end{array}$ & $\begin{array}{c}\text { BBS } \\
(0-56 \mathrm{pts}) \\
\end{array}$ & $\begin{array}{l}\text { FGA } \\
(\mathrm{pts}) \\
\end{array}$ \\
\hline 1 & $M$ & 33 & 9 & 18 & 45 & 14 \\
\hline 2 & M & 26 & 5 & 18 & 50 & 20 \\
\hline 3 & F & 45 & 21 & 12 & 54 & 26 \\
\hline 4 & M & 42 & 17 & 6 & 48 & 18 \\
\hline 5 & F & 55 & 6 & 6 & 52 & 24 \\
\hline 6 & $\mathrm{~F}$ & 36 & 6 & 5 & 54 & 27 \\
\hline 7 & F & 42 & 19 & 5 & 53 & 26 \\
\hline 8 & M & 44 & 9 & 2 & 54 & 26 \\
\hline 9 & M & 63 & 7 & 5 & 54 & 25 \\
\hline 10 & $\mathrm{M}$ & 66 & 4 & 2 & 46 & 22 \\
\hline
\end{tabular}

\subsection{Experimental Conditions and Data Collection}

Participants walked for 12-m in the laboratory under two different conditions: (1) at their preferred walking pace and stride length (Preferred), and (2) with a narrow base of support by placing their next foot directly in front of the other (Narrow). Instructions were to walk in a straight line while carefully placing one foot directly in front of the other although there was no requirement that the feet would touch when on the ground. Encouragement was given to be comfortable while walking for each condition and condition order was randomised across participants.

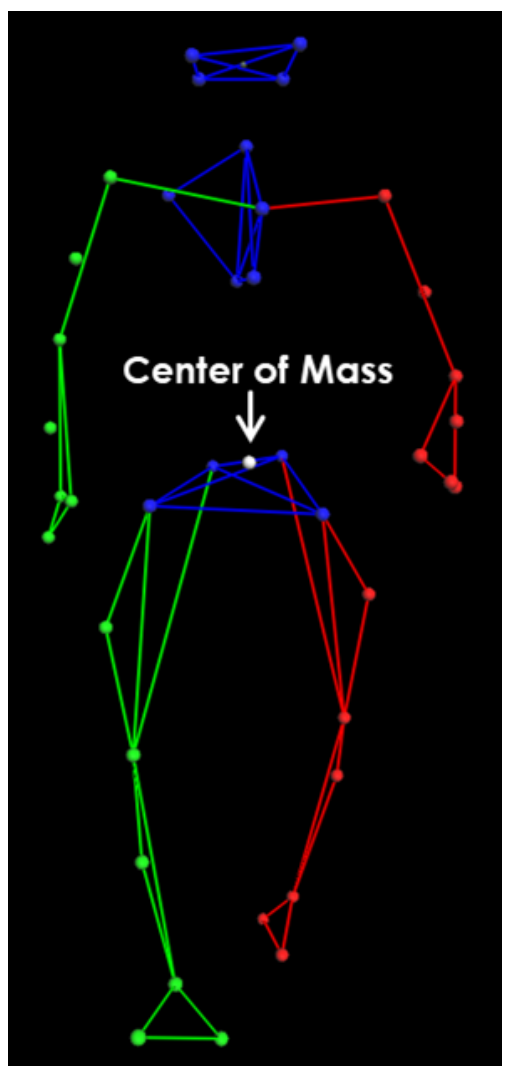

Figure 1. Frontal plane participant center of mass (COM) calculated from marker data (Vicon plug-in gait) and anthropometrics
Motion of 39 retro-reflective markers $(10 \mathrm{~mm}$ diameter, B\&L Engineering, Santa Ana, CA, USA) attached to the body landmarks was recorded with Vicon (12 camera, T160 system, 100Hz) (Figure 1). The standard Vicon plug-in-gait model with bilateral posterior superior iliac spine markers was used to guide marker location. Marker data were post-processed (Vicon Nexus 2.5) to ensure accurate identification and any gaps, which were minimal, were filled. Gait events corresponding to contact with the toe on/off ground were visually identified and marked within Nexus. Body COM during each trial was calculated from marker data and participant anthropometry recorded prior to experimental conditions. The COM data were then differenced to diminish effects of uneven floor plane and minor changes in walking direction. Because walking progression is mostly in forward direction and causes non-stationarity of $\mathrm{COM}$ data in anterior-posterior direction, and this study concerned how movement varies as related to stability, only frontal plane (vertical and lateral) differenced two-dimensional COM coordinates were submitted to RQA (Figure 1).

\subsection{Recurrence Quantification and Statistical Analyses}

Differenced frontal plane COM data were cropped to 6 mid-trial steps to minimise starting and stopping effects which could cause non-stationarity in the data. As the utility of nonlinear techniques such as RQA is to describe how the COM waveform varies, in accordance with standard practices [11], the data for each trial were normalized to eliminate dependencies and minimize sensitivities to temporal (e.g. speed of walking) and amplitude differences between trials and conditions. Normalization consisted of resampling the cropped data so each trial was the same number of frames $(n=1000)$ [12], and then the resampled data were Z-score normalized prior to RQA (Figure 2, top row).

RQA was performed with the CRP Toolbox suite (version 5.19) [19]. As a first step in RQA, all trials are typically screened to determine appropriate embedding parameters which include time lag, embedding dimension 
and neighborhood threshold for reconstructing the phase space [9]. RQA results can be sensitive to embedding parameters, especially time lag and dimension [20, 21]. Data sets, and experimental conditions within, may be affected differently by noise and other deterministic and stochastic factors, thus confounding the process of determining appropriate embedding parameters with standard techniques (e.g. first zero-crossing of autocorrelation and false nearest neighbors for time lag and embedding dimension, respectively). In this study, to avoid dependency on embedding parameters and any confusion regarding dependency, thus simplifying the analysis and interpretation, phase spaces were constructed from the unembedded frontal plane differenced, resampled, Z-score normalized COM data, i.e. no time lag or embedding dimension (Figure 2, middle row).
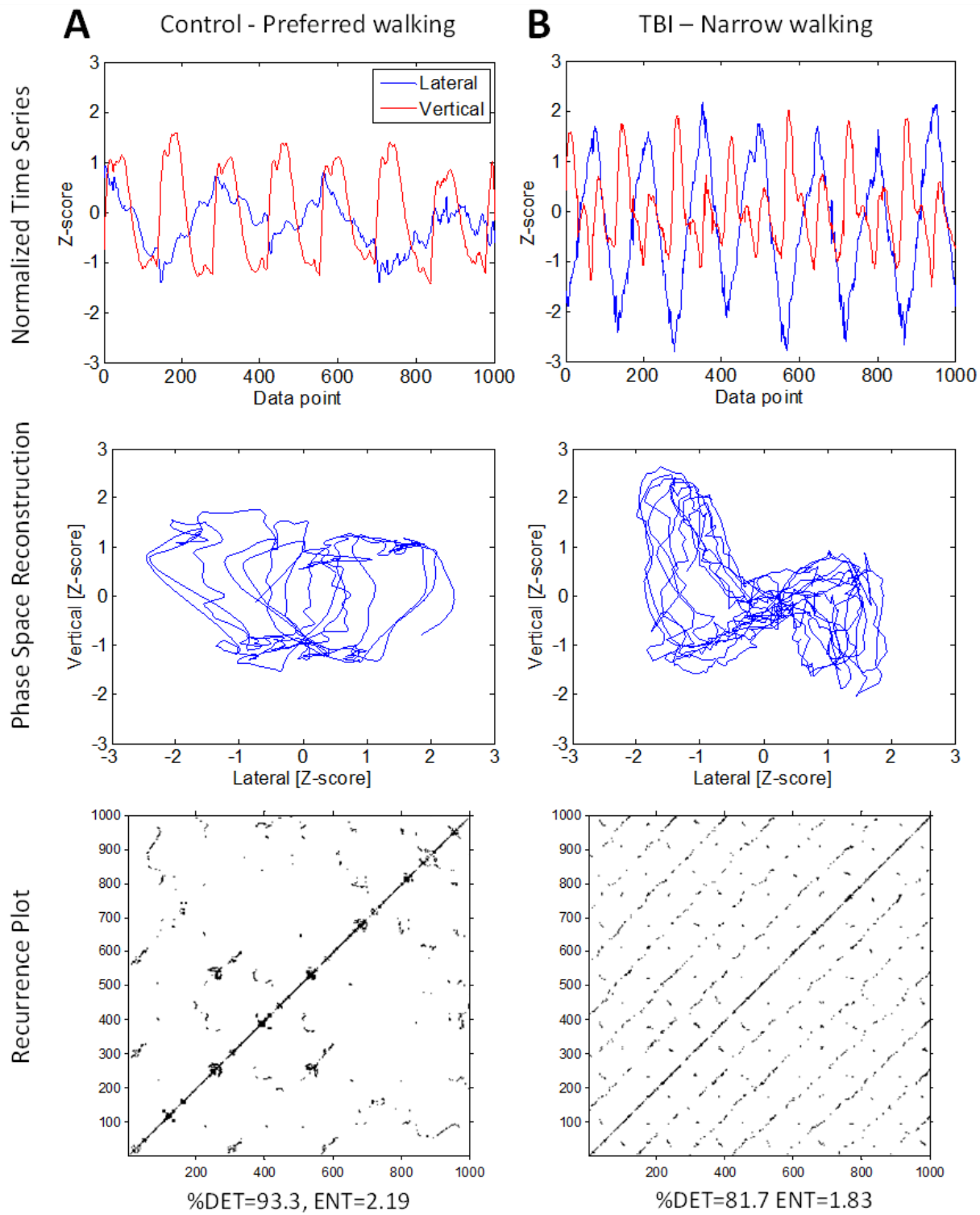

Figure 2. Frontal plane participant center of mass (COM) calculated from marker data (Vicon plug-in gait) and anthropometrics. RQA for typical front plane center-of-mass (COM) waveform data. (A) Control group preferred walking and (B) TBI group narrow walking. Top row: Z-score normalized (i.e standard deviation) for 6 walking steps, time normalized to 1000 data points. Middle row: Phase space reconstruction of Z-score normalized data. Bottom row: Recurrence plots $(\mathrm{RP})$ for phase space with fixed recurrence rate $(\% \mathrm{REC}=2 \%)$ and measures for \%DET and ENT below recurrence plots 
From the phase spaces, recurrence plots (RPs) were constructed using a flexible neighborhood radius to ensure a fixed recurrence rate $(\%$ REC $=2 \%)$ [22] (Figure 2, bottom row). This approach was chosen to prevent over-saturation of RPs [23] while ensuring sufficient information was extracted from each waveform, i.e. avoiding under-saturation, to allow comparison across conditions and between the two groups (Preferred and Narrow). The distance matrix was rescaled based on the maximum normalized distance. The patterns of darkened pixels allow for the measurement of recurrence properties and provide information on waveform predictability and complexity.

A greater number of darkened points in the RP is measured as higher recurrence (\%REC). The percentage of recurrent points, here $\geq 2$ points, forming diagonal lines measures \%DET. Together, greater \%REC and \%DET indicate greater regularity and predictability over consecutive samples of walking during the trial [24]. Periodic systems have greater deterministic recurrence, leading to RPs for these systems having longer diagonal lines and a greater number of lines. Here, since \%REC was fixed across all trials, it was eliminated from interpretation leaving \%DET as a sole measure of predictability. Increased complexity in the distribution of diagonal line lengths indicates greater Shannon Entropy and illustrates increased system complexity [23]. While RQA can be used to measure other non-linear properties, the decision was to focus on \%DET and Entropy as this was an initial study of predictability and complexity of walking data as affected by a simple intervention of walking with narrow base of support and for a relatively small number of subjects. For each RQA measure, 2-way ANOVAs (Preferred and Narrow by TBI and Control) were performed $(\alpha=0.05)$. When significant differences were detected for main effects, paired t-tests were applied to test for differences between conditions or groups with Bonferroni adjustment to guard against Type I errors.

\section{Results}

There were significant differences in \%DET $(\mathrm{p}<0.0001)$ when walking with preferred compared to narrow, but no difference was found in \%DET between Control and TBI groups $(p=0.17)$ (Figure 3A). Within the Control group, \%DET was significantly reduced $(p=0.0019)$ for narrow $(81.5 \pm 2.3 \%$, mean $\pm \mathrm{SE})$ compared to preferred $(91.9 \pm 1.3 \%)$ walking. Likewise, for the TBI group \%DET was significantly reduced for narrow $(77.5 \pm 2.9 \%)$ compared to preferred $(90.2 \pm 0.9 \%)$ walking $(\mathrm{p}=0.0008)$.

Significant differences were found for Entropy $(\mathrm{p}=0.0001)$ for preferred compared to a narrow walking, but no difference was between Control and TBI groups in Entropy $(\mathrm{p}=0.33)$ (Figure 3B). For the Control group, there was a tendency toward a decrease in in Entropy when walking narrow compared to preferred $(1.75 \pm 0.07$ bits and $2.05 \pm 0.08$ bits, respectively) but the difference was not significant $(\mathrm{p}=0.0142)$. While for the TBI group, there was a significant decrease $(\mathrm{p}=0.0038)$ in Entropy when walking narrow $(1.65 \pm 0.08$ bits $)$ compared to preferred $(2.00 \pm 0.05$ bits $)$.
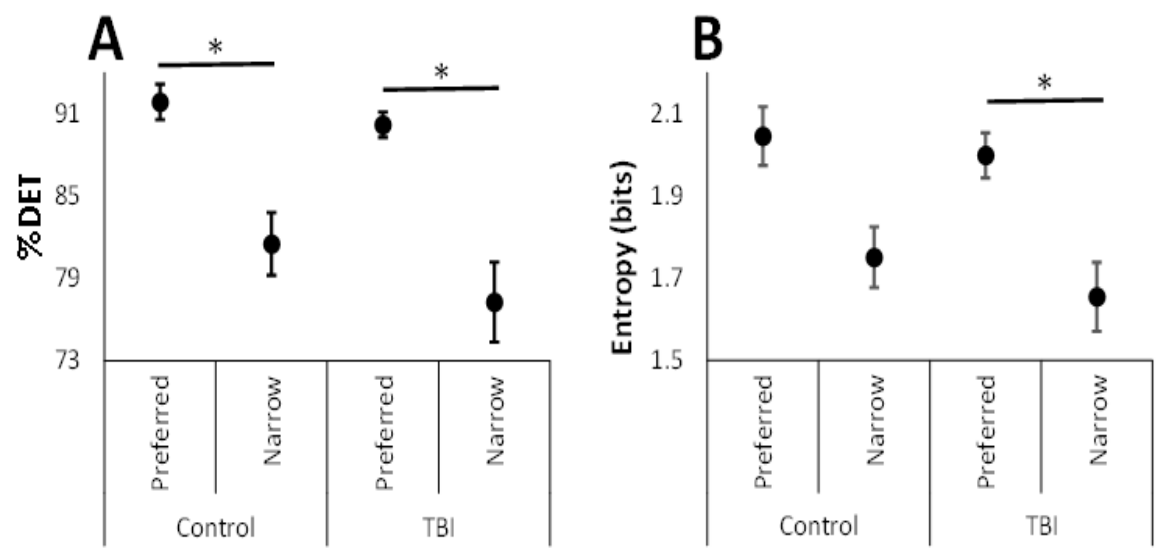

Figure 3. Percent Determinism (\%DET) and Entropy (bits) for TBI and Control groups in preferred and narrow walking conditions (mean and standard error). *Significant differences ( $<<0.0042$, Bonferroni correction) 


\section{Discussion}

This study evaluated the predictability and complexity of COM motion under preferred and narrow walking conditions for TBI and age-matched control subjects in order to determine if non-linear measures may be appropriate for identifying differences between these two groups. The results for \%DET indicate that walking with a narrow base of support results in a less predictable COM motion and gait of less regularity than preferred walking in both the Control and TBI groups. Thus, the first hypothesis of this study, that TBI was expected to result in less predictable COM motions during narrow walking and compared to Control participants, was partially supported. The differences in nonlinear measures of predictability, \%DET, were detected between preferred and narrow walking, but not between control participants and participants with TBI. Decreased predictability of the COM sway during challenging narrow walking could be interpreted as a decreased ability of the nervous system to use synergetic and already established patterns of multi-muscle and inter-joint interaction for controlling stability. Instead, the system may follow the route of "improvising", developing new and/or adjusting postural patterns that are less predictable and more variable, as all motions that have been practiced for the first time. In this regard, the main question is which criterion the system uses to produce this adaptation. If this criterion is minimization of COM oscillations, then when applied to postural stability adjustments, the adaptations may not be easily detectable visually or even by such sophisticated techniques as motion capture. In other words, postural adjustments and adaptations will compensate and make posture appear stable during narrow walking. However, these compensations will not prevent postural stability from being less responsive to even small perturbations, and therefore both less predictable and controlled, respectively. These effects have been observed in different populations of subjects [25-27]. Postural adaptation could be considered as masking postural instability, but not making the posture more stable. Thus, detecting these hidden postural challenges and especially differentiating them from actually stabilizing factors is important. This detection is possible by exploring and testing alternative methods to kinematic standards measurement techniques, such as RQA, that has been shown to be are more sensitive tool for detecting postural deviations.

Analogous to the first finding, the second finding of the present study, the decrease in Entropy demonstrates that narrow walking causes COM motion to be of reduced complexity in TBI and control subjects with no difference between groups. Motion complexity can somehow reflect a number of degrees of freedom employed by the system to control postural stability. Decreased complexity could be interpreted as fewer degrees of freedom, while the increased number would indicate a greater involvement of segments and angular displacements in postural control. If using this analogy, the results of our study are consistent with ideas introduced by N. Bernstein [28]. He suggested that while experiencing a challenging situation, learning a new movement, having some deficiency a person tends to "freeze" his body. Freezing in this context means an attempt to minimize movement complexity. Although this phenomenon was first introduced by Bernstein, it has been confirmed in many other studies of healthy individuals and those with motor problems due to neurological disease and aging [29]. Muscle co-contraction immobilizing motions at certain joints could achieve reducing complexity. While such a strategy can be effective during quiet standing, it limits the ability of the system to effectively respond to perturbation during activities that requires dynamic stability such as walking and especially when challenged by environments and tasks. Thus, reduced complexity of the COM motion in our participants during narrow walking provides another evidence of efficiency of RQA in detecting gait deviations that other standard parameters and measures typically fail to detect.

\section{Conclusions and Limitations}

In summary, the present study revealed decreased predictability and complexity of the COM oscillations in individuals with TBI and Control subjects during challenging conditions when compared to normal walking. It is important to emphasize that these gait deviations were not detected by the use of other kinetic and kinematic measures as performed in previous studies, but become recognized only with the use of RQA. Thus, RQA showed potential to become a valid and reliable measurement technique and/or predictor of gait deviations, and could be utilized in future studies.

\section{REFERENCES}

[1] P.C. Fino, L. Parrington, W. Pitt, D.N. Martini, J.C. Chesnutt, L.-S. Chou, L.A. King. Detecting gait abnormalities after concussion or mild traumatic brain injury: A systematic review of single-task, dual-task, and complex gait. Gait and Posture, 62, 157-166, 2018.

[2] G. Williams, M.E. Morris, A. Schache, P.R. McCory. Incidence of gait abnormalities after traumatic brain injury. Arch Phys Med Rehab, 90, 587-593, 2009.

[3] H.M. Austin, N. Balendra, J.E. Langenderfer, K.I. Ustinova. Decomposition of leg movements during overground walking in individuals with traumatic brain injury. Brain Injury, 32, 739-746, 2018.

[4] D. Vaillancourt, K.M. Newell. Changing complexity in human behavior and physiology through aging and disease. 
Neurobio Aging, 23, 1-11, 2002.

[5] S. Morrison, K.M. Newell. Aging, neuromuscular decline, and the change in physiological and behavioral complexity of upper-limb movement dynamics. J. Aging Res, 2012, 891218, 2012.

[6] P.C. Fino, M.A, Nussbaum, P.G. Brolinson. Decreased high-frequency center-of-pressure complexity in recently concussed asymptomatic athletes. Gait and Posture, 50, 69-74, 2016.

[7] N. Stergiou, L.M. Decker. Human movement variability, nonlinear dynamics, and pathology: Is there a connection? Hum Move Sci, 30, 869-888, 2011.

[8] J.T. Cavanaugh, K.M. Guskiewicz, N. Stergiou. A Nonlinear Dynamic Approach for Evaluating Postural Control: New Directions for the Management of Sport-Related Cerebral Concussion. Sports Med, 35, 935-950, 2005.

[9] M.A. Riley, R. Balasubramaniam, M.T. Turvey. Recurrence quantification analysis of postural fluctuations, Gait and Posture, 9, 65-78, 1999.

[10] J.M. Schmitt, D.L. Regis, M.A. Riley. Dynamic patterns of postural sway in ballet dancers and track athletes. Exp Brain Res, 163, 3, 370-378, 2005.

[11] A.O. Assis, F.B. Rodrigues, A. Carafinia, T.S. Lemes, G.A.G. de Villaa, A. Andrade, M.F. Vieiraa, Influence of sampling frequency and number of strides on recurrence quantifiers extracted from gait data. Comp Bio Med, 119, 103673, 2020.

[12] F.S. Labini, A. Meli, Y.P. Ivanenko, D. Tufarelli. Recurrence quantification analysis of gait in normal and hypovestibular subjects. Gait and Posture, 35, 48-55, 2012.

[13] K. Li, Z.M. Li. Cross recurrence quantification analysis of precision grip following peripheral median nerve block. J. NeuroEngineering Rehab, 10, 2013.

[14] H. Negahban, M. Salavati, M. Mazaheri, M.A. Sanjari, M.R. Hadian, M. Parnianpour. Non-linear dynamical features of center of pressure extracted by recurrence quantification analysis in people with unilateral anterior cruciate ligament injury. Gait and Posture, 31, 450-455, 2010.

[15] B. Mason, K. Page, K. Fallon. An analysis of movement and discomfort of female breast during exercise and the effects of breast support in three cases. J Sci Med Sport, 2, 134-144, 1999. T. Klockgether, G. Schroth, C.H. Diener, J. Dichgans, Idiopathic cerebellar ataxia of late onset: natural history and MRI morphology. J Neurology, Neurosurgery and Psychiatry, 53, 297-305, 1990.

[16] K. Berg. Measuring balance in the elderly: Preliminary development of an instrument. Physiotherapy Canada, 41,
304-311, 1989

[17] D.M. Wrisley, G.F. Marchetti, D.K. Kuharsky, S.L. Whitney, Reliability, internal consistency, and validity of data obtained with the functional gait assessment. Phys Ther, 84, 906-918, 2004.

[18] Management of Concussion/Mild Traumatic Brain Injury. VA/DoD Clinical Practice Guidelines. 2016.

[19] N. Marwan, N. Wessel, N., U. Meyerfeldt, A. Schirdewan, J. Kurths, J. Recurrence-plot-based measures of complexity and their application to heart-rate-variability data. Phys Rev E Statis, Nonlin, Biol, and Soft Matter Phys, 66, 026702, 2002.

[20] N. Marwan. How to avoid potential pitfalls in recurrence plot based data analysis. Intern J Bifurcation and Chaos, 21, 1003-1017, 2011.

[21] S. Ramdani, G. Tallon, P.L. Bernard, H. Blain. Recurrence quantification analysis of human postural fluctuations in older fallers and non-fallers. Ann Biomed Engin, 41, 1713-1725, 2013.

[22] E.S. Jackson, M. Tiede, M. A. Riley, D.H. Whalen Recurrence quantification analysis of sentence-level speech kinematics. J Speech, Lang, and Hear Res, 59, 1315-1326, 2016.

[23] S. Bonnette, J.A. Diekfuss, D. Grooms, G.D. Myer, W.P. Meehan, D.R. Howell. Integrated linear and nonlinear trunk dynamics identify residual concussion deficits. Neurosci Letters, 729, 134975, 2020.

[24] C.A.K. Kwuimy, S.A. Adewusi, C. Nataraj. Characterization of the vibration transmitted in the human arm using selected recurrence quantification parameters. Nonlin Dyn, 88, 2873-2887, 2017.

[25] A.H. Vette, D.G. Sayenko, M. Jones, M., M.O. Abe, K. Nakazawa, K. Masani. Ankle muscle co-contractions during quiet standing are associated with decreased postural steadiness in the elderly. Gait and Posture, 55, 31-36, 2017.

[26] S. Tassani, J.M. Font-Llagunes, M.A. González Ballester, J. Noailly. Muscular tension significantly affects stability in standing posture. Gait and Posture, 68, 220-226, 2019.

[27] R. Kitatani, K. Ohata, Y. Hashiguchi, K. Sakuma, N. Yamakami, S. Yamada. Clinical factors associated with ankle muscle coactivation during gait in adults after stroke, NeuroRehabilitation, 38, 351-357, 2016.

[28] N. Bernstein. The coordination and regulation of movement. Pergamon Press, 1967.

[29] A. Shumway-Cooke, M.H. Woollacott, Motor Control: Translating Research into Clinical Practice. 5th ed, Wolters Kluwer Health/Lippincott Williams \& Wilkins, 2017. 